Network project (EEN) has been created in 2008 with the support of the European Commission (DG Healthand Consumers), private partners and the involvement of four European university teams. To be run until 2011, the EEN has been designed to enrich the EPODE methodology on its four pillars: involvement of political representatives, scientific evaluation and dissemination, methods and social marketing and public-private partnership.

Poster Abstracts: Prevalence and Body Composition 01 - The analysis of schoolchildren growth development

\author{
VI Velychko', IV Saryłskyi ${ }^{2}$, IL Babiy' and LI Zasypka'
}

'Department of Pediatrics, Odessa National Medical University, Odessa, Ukraine: ${ }^{2}$ Odessa Medical Institute of International Humanitarian University, Odessa, Ukraine

Introduction: The rate of physical growth development is an important criterion for the complex assessment of health of children and adolescents. Heredity, environment, work and study conditions, nutrition and physical activity influence upon the growth development of schoolchildren.

Method: In all, 1386 schoolchildren aged between 6 and 16 years were examined: 714 boys and 672 girls. The harmonic physical growth development was determined amongst 1386 (68.3\%) of examined children. Disharmonic development with the acceleration of biological development was determined amongst 336 (16.5\%) schoolchildren whereas the deceleration of biological development was found amongst $132(6.5 \%)$ children. Sharply disharmonic physical growth development was detected in $176(8 \cdot 7 \%)$ cases where sixty-four $(3 \cdot 2 \%)$ children have manifested deceleration of the biological development and 112 (5.5\%) have accelerated development.
Results: The harmonic growth development was observed for $66 \%$ or more examined children only for the age 9-15 years. Only $32 \%$ of 7 -year-old children and $22 \%$ of 8 -yearold children have harmonic growth development. There were $47 \%$ and $53 \%$ of harmonically developed individuals in the groups of 6- and 16-year-old children correspondingly. The highest rate of disharmonic physical growth development was characteristic for 6-year-old children $-41 \cdot 2 \%$ (due to excessive fat deposits $-29 \cdot 4 \%$, due to the deficiency of weight $-11 \cdot 8 \%$ ). Children aged 7 years have disharmonic growth development in $35.7 \%$ cases, where the excessive body weight is detected for $28.6 \%$, and deficient weight - for $7 \cdot 1 \%$. Thus the main reason for disharmonic growth development was the excessive fat deposits and only the group of 9-year-old children had more cases of the deficient weight.

Conclusions: Described anomalies of the physical growth development could be related to the unfavourable impact of the environmental factors.

\title{
02 - Severe obesity in Italian children and related factors: data from Okkio alla Salute national survey
}

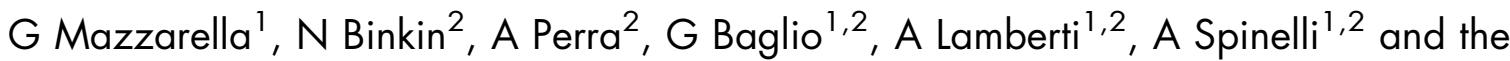 \\ Technical Committee of OKkio alla Salute
}

'OKkio alla Salute, Technical Committee, National Institute of Health, Rome, Italy: ${ }^{2}$ National Centre for Epidemiology, Surveillance and Health Promotion, National Institute of Health, Rome, Italy

Introduction: To estimate the prevalence of childhood obesity in Italy a nationwide representative survey among third-grade students was carried out in 2008.

Method: Study population included all children aged 8-9 years whose parents agreed to opt-out consent. The sampling unit was each class and cluster sampling identified classes for participation. Questionnaires were completed by children, parents and teachers. Children's weight and height were obtained by well-trained staff using standardized equipment. WHO age- and sex-specific 